\title{
Monitoring changes in skin temperature associated with exercise in horses on a water treadmill by use of infrared thermography
}

\author{
Kelly Yarnell ${ }^{\mathrm{a}, *}$, Jennifer Fleming ${ }^{\mathrm{b}}$, Tim D. Stratton ${ }^{\mathrm{a}}$, Rebecca Brassington ${ }^{\mathrm{b}}$ \\ a Nottingham Trent University, Southwell, Nottingham NG25 OQF, United Kingdom \\ ${ }^{\mathrm{b}}$ Bishop Burton College, Beverley, East Yorkshire HU17 8QG, United Kingdom
}

\section{A R T I C L E I N F O}

\section{Article history:}

Received 5 June 2014

Received in revised form

13 August 2014

Accepted 13 August 2014

Available online 19 August 2014

Keywords:

Infrared thermography

Equine

Surface temperature

\begin{abstract}
A B S T R A C T
Infrared thermography (IRT) was used to assess surface temperature change as an indirect measure of muscle activity and exercise associated changes in blood flow in the working hind limb muscles of horses $(n=7)$ undergoing water treadmill exercise. Three treatments were investigated including the treadmill ran dry (TD), water at the height of the proximal interphalangeal joint (PIP) and water at the height of the carpus (CP). Maximum skin surface temperature was recorded from the region of the semitendinosus muscle during exercise at each water height. There was a significant difference in surface hind limb temperature between exercise on the water treadmill ran dry and with water at the height of the PIP and CP $(P<0.0001)$ with hotter temperatures recorded during the TD treatment. There was a greater increase in surface temperature of the hind limbs from pre exercise to maximum temperature during the PIP and CP treatments when compared to the TD treatment, however, this was not significant $(P=0.58)$. There was no significant difference in surface hind limb temperature found between exercise in water at the height of the PIP and water at the height of the CP. The findings from this study suggest that IRT is able to non-invasively detect muscle activity and associated changes in blood flow whilst horses are exercised on a water treadmill. IRT could potentially be used as an alternative method to assess muscle activity and temperature change in an aquatic environment where existing methods present methodological challenges.
\end{abstract}

(c) 2014 Elsevier Ltd. All rights reserved.

\section{Introduction}

Water treadmills are a method of hydrotherapy used to improve the cardiovascular and muscular capacities of horses (Tokuriki et al., 1999) and they are becoming increasingly popular in training centres worldwide, despite little being known about their physiological effects. Exercise in water encourages muscle development, develops muscle tone and improves muscle strength (King et al., 2012) with a reduction in the concussive forces and weight bearing stresses experienced by the equine distal limb during land based exercise (Miyoshi et al., 2004).

Both elite and non-elite sport horses suffer with repetitive strain injuries to the hind limb muscle groups, particularly semitendinosus muscle strain (McKenzie, 2005; Rivero and Piercy, 2008) which requires rehabilitation in order for the horse to return to competition fitness. The muscles of the hind limb provide the horse with the propulsive forces required in many equestrian sporting disciplines. The hip retractors (gluteus medius

\footnotetext{
* Corresponding author.

E-mail address: kelly.yarnell@NTU.ac.uk (K. Yarnell).
}

and biceps femoris) are the primary muscles responsible for providing power to the horse (Crook et al., 2010). The biceps femoris and semitendinosus muscles combine to form the equine hamstring muscle group which is fundamental in facilitating locomotion (Pusey et al., 2011)

Water treadmill exercise may be useful for horses undergoing rehabilitation from injury to the limbs and back (Scott et al., 2010). The water provides buoyancy and assists the horse in lifting its limbs in the vertical plane and provides resistance to the limbs in the sagittal plane (King et al., 2012) whilst the treadmill allows speed to be controlled. A feature of many water treadmills is the ability to alter the water height in which the horse is exercising in and it has been shown that different water heights are responsible for biomechanical alterations in gait with Scott et al. (2010) reporting that horses walking in water at the level of the carpus resulted in a lower stride frequency compared to walking in water at hoof height. The biomechanical changes may be due to a higher stepping motion from the horse in an attempt to minimise resistance of the water on the distal limb. This could potentially result in greater activity of the muscles involved in flexion of the hind limb including the semitendinosus. These findings suggest that there is greater resistance placed upon the horse when water 
is higher up the limb which has important implications for rehabilitation and training regimes of horses using the water treadmill. A study by Robert et al. (2000) used electromyography (EMG) to determine how hind limb muscle activity varied with speed and incline in the horse on a land based treadmill. The study concentrated on the gluteus medius and tensor fasciae latae muscles and concluded that both increases in speed and incline lead to an increase in electromyographic activity and hence a higher workload for the muscles investigated.

It is important to develop an understanding of the physiological response of muscles during exercise in water in order to determine the most appropriate programme and workload for targeting specific muscle groups. No research has been carried out to date on muscle activity at different water heights whilst exercising on the water treadmill due to the assessment of muscle activity during aquatic exercise presenting methodological challenges. Electromyography (EMG) provides an assessment of muscle activity; however, the equipment presents challenges when testing in water due to the presence of electrical wiring and self-adhesive electrodes.

A solution to this problem may be the assessment of surface temperature using infrared thermography (IRT) as an indirect measure of the underlying physiological response to exercise. Considerable heat is produced during muscle contraction (Shepherd, 1982). Positive correlations have been found between muscle temperature and exercise duration in horses, with muscle temperature gradually increasing as exercise continues up to a mean temperature of $38.8( \pm 0.5)^{\circ} \mathrm{C}$ measured by muscle biopsy (Lovell et al., 2006).

Infrared thermography can identify changes in skin surface temperature which has been found to directly reflect the underlying circulation, tissue metabolism and local blood flow (Eddy et al., 2001; Tunley and Henson, 2004). Previous work has shown that water treadmill exercise is predominantly an aerobic activity (Voss et al., 2002; Linder et al., 2003) and the overall effect of aerobic exercise on circulation is to increase blood flow to exercising muscles in order to meet the metabolic demands of the working tissues (Van de Graaffe et al., 1999). As a result of these physiological changes in blood flow and muscle contraction, radiated heat will increase and can be identified using IRT.

IRT was used to assess muscle temperature in the human arm during wheelchair driving (Matsuo et al., 2006). Surface temperature was found to significantly increase in the lower arm which provides the power for the activity with a minimal increase in surface temperature in the upper arm that plays a minor role in the activity. In addition, electromyogram measurement showed higher electrical activity of the muscle in the lower arm when compared to the upper arm. These findings indicate that thermography can be a useful tool to assess radiated heat as a noninvasive measure of muscle activity and its use in other species warrants investigation.

IRT is a commonly used tool in many fields of science; however, its application in the equine industry has largely been in the field of veterinary diagnostics (Von Schweinitz, 1999; Turner, 2001) and it has not been until recently that wider applications have been considered. Recent applications of IRT in horses are the assessment of surface temperature change during exercise (Simon et al., 2006) and temperature change of the eye associated with the physiological stress response (McGreevy et al., 2012; Yarnell et al., 2013). Interest is growing regarding further applications and uses of thermal imaging due to its non-invasive nature and ability to rapidly assess temperature, both of which are advantageous when working with horses. The use of IRT to monitor surface temperature change associated with muscle contraction and alterations in associated blood flow on a water treadmill has not yet been explored.
Traditional methods of monitoring temperature in horses have their own limitations including handling and manipulation of the horse and invasive surgical implantation of biotelemetry equipment (Parrott et al., 1999). External sensors can act as insulators that may confound results and solid probes can give false readings due to disruption by hair fibres (Nakayama et al., 2005). There are factors which must be considered when utilising IRT to assess the surface temperature of animals including fluctuations in ambient temperature and airflow, radiation from sunlight particularly in animals of varying coat colour (Cena and Clark, 1973) and a moist or dirty coat of the animal (Tunley and Henson, 2004). Therefore, measures must be taken to minimise sudden changes in ambient temperature in addition to capturing images in a suitable environment free from draughts or powerful solar light and with a clean, dry horse (Schaefer et al., 2002).

The skin overlying muscles is subject to an increase in temperature during muscular activity (Redaelli et al., 2014). Infrared thermography offers a non-invasive method to assess this increase in surface temperature and overcomes the methodological challenges faced when using existing methods of temperature assessment of horses in an aquatic environment.

IRT was utilised during this study to assess the effects of differing water heights on the surface temperature of the hind limbs in the region of the semitendinosus muscle. The muscles in this area play an important role in equine locomotion and are often targeted in rehabilitation and training programmes that utilise a water treadmill and the semitendinosus muscle lies superficially to the skin and can be palpated by hand. Horses were exercised with the treadmill ran dry (TD), with water at the height of the proximal interphalangeal joint (PIP) and with water at the height of the carpus (CP). Recent research has demonstrated that when horses were exercised in water at each of these heights, differences in biomechanical parameters were found (Scott et al., 2010), therefore, we wished to investigate if the same were true for muscle activity and whether this can be identified using IRT.

Until now no attempt has been made to assess muscle activity and associated changes in blood flow during exercise on a water treadmill despite the importance of developing an understanding of how the addition of water at different heights alters these parameters.

\section{Materials and methods}

\subsection{Horses and husbandry}

Horses $(n=8)$ were provided by Bishop Burton College Equestrian Centre. Horses had a mean age of 14 years ( \pm 5 years) and a mean height of $156.1 \mathrm{~cm}( \pm 8 \mathrm{~cm})$. Mean PIP height was $11.7 \mathrm{~cm}$ $( \pm 1.4 \mathrm{~cm})$ and mean $\mathrm{CP}$ height was $44 \mathrm{~cm}( \pm 3 \mathrm{~cm})$. The group consisted of five geldings and three mares and all horses were examined and declared sound by the college vet prior to the study commencing. Ethical approval was gained from Bishop Burton college ethics committee. All horses had resided at the college for at least one year prior to the study and were ridden for up to two hours per day, six days per week. All horses were involved in practical lessons that utilised the water treadmill throughout the academic year (twelve week term $=$ mean $8 \pm 1.7$ sessions per horse), therefore, they were all familiar with the process and surroundings of the water treadmill building.

\subsection{Experimental design}

The study was carried out over three consecutive days in November 2012. Each horse was lead individually from their stable to the building that housed the water treadmill (Aqua Line, 


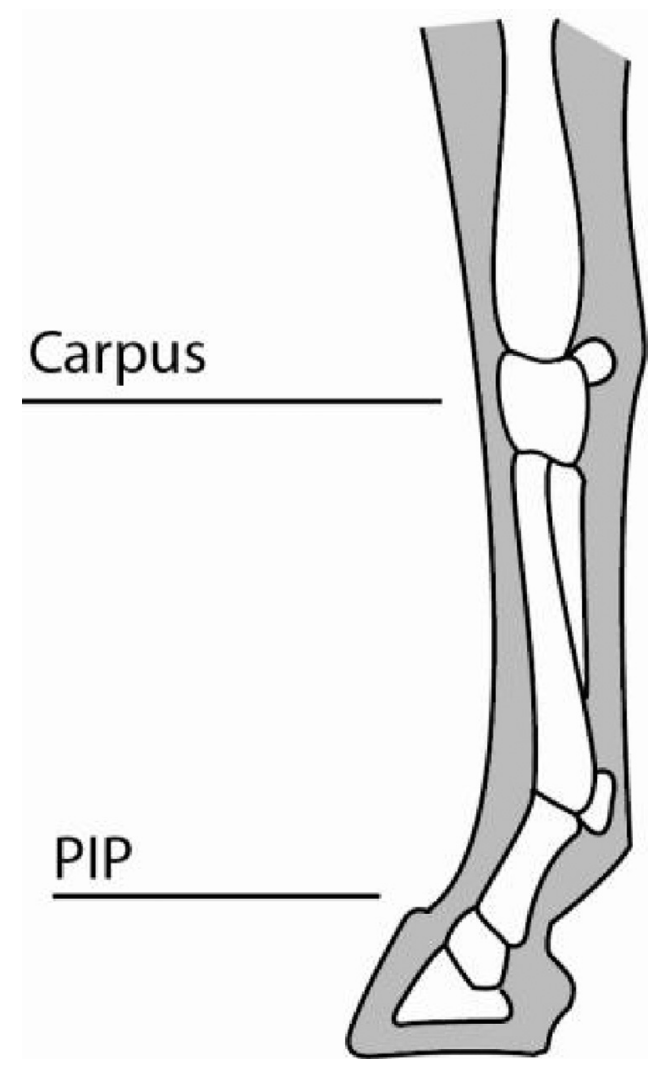

Fig. 1. The equine limb showing the water heights used during the study. This image shows the equine limb and the levels of water height used in this study which were the proximal interphalangeal joint (PIP) and the carpal joint (CP). Horses $(n=8)$ were exercised at each height in addition to the treadmill ran dry (TD) on three separate occasions.

ACTIVO-MED water treadmill, Mechtersen, Germany). The distance from the stable to the treadmill room was dependant on the location of the individuals stable with a mean distance of $120 \mathrm{~m}$ $( \pm 25 \mathrm{~m})$. Horses were exercised on the water treadmill in their own bridle with a rein held by the same familiar handler. Each horse was tested at three water heights (Fig. 1) which were (1) water treadmill ran dry to simulate a standard land treadmill (TD), (2) water at the height of the proximal interphalangeal joint (PIP) and (3) water at the height of the carpus (CP). This allowed comparisons in surface hind limb temperature to be made between the three exercise protocols.

Each horse was exercised for $15 \mathrm{~min}$ at each water height. The speed of the treadmill belt varied dependant on the individual horse. Each horse was assigned a comfortable active walking speed with the mean walking speed for all horses being $5.7( \pm 0.3) \mathrm{kph}$. During the first $3 \mathrm{~min}$ of water treadmill exercise the water level was increased to the desired level. The mean time taken for water to reach the height of the PIP was $70 \mathrm{~s}( \pm 6 \mathrm{~s})$ and the mean time taken for water to reach the height of the CP was $180 \mathrm{~s}( \pm 6 \mathrm{~s})$. During the final $3 \mathrm{~min}$ of water treadmill exercise, the water level was drained. These periods of time were utilised as the warm up and cool down periods for the horses. Exercise from $3 \mathrm{~min}$ to 12 min involved the horse exercising at the desired water height (TD, PIP or CP). Water in the treadmill was not artificially warmed or cooled in any way and was stored at room temperature. Ambient temperature was monitored in the treadmill room (Lascar USB-2 temperature data logger, UK) throughout the study. Mean ambient temperature was $9.7( \pm 0.6){ }^{\circ} \mathrm{C}$ with no large fluctuations recorded during the testing period. There was no air frost on any of the study days and no precipitation. Horses were tested in the
Table 1

The ten IRT sampling time points during each treadmill session. This table details the ten sampling time points when IRT was utilised to assess surface temperature of the hind limbs in the study horses. The image number is given along with details of the timing of image capture from six minutes pre exercise, 15 min during exercise and until three minutes post-exercise.

\begin{tabular}{ll}
\hline Image number & Details of timing of thermal image capture \\
\hline 1 & $\begin{array}{l}6 \text { min before commencing water treadmill exercise (in stable) } \\
3 \text { min before commencing water treadmill exercise (in water } \\
\text { treadmill building) }\end{array}$ \\
3 & $\begin{array}{l}\text { Immediately before commencing exercise (standing on } \\
\text { treadmill stationary) }\end{array}$ \\
4 & 3 min from commencing water treadmill exercise (warmup) \\
5 & 6 min from commencing water treadmill exercise \\
6 & 9 min from commencing water treadmill exercise \\
7 & 12 min from commencing water treadmill exercise \\
8 & 15 min from commencing water treadmill exercise, stationary \\
9 & Immediately post-exercise (18 min from commencing \\
10 & exercise. Horse stationary) \\
& Three minutes post-exercise (21 min from commencing \\
& exercise. Horse Stationary)
\end{tabular}

same order on three separate consecutive days with one day used for each of the water heights.

\subsection{Thermal data collection}

Thermal images were captured at 10 separate sampling time points during each horse's water treadmill session (Table 1). All of the thermal images that were collected throughout this study were obtained using a FLIR B335 $(30 \mathrm{~Hz}, 2010$ model $)$ thermal imaging camera. The camera has a thermal sensitivity of $<0.05^{\circ} \mathrm{C}$ at temperatures of $\geq 30^{\circ} \mathrm{C}$, with a range of temperature detection between $-20{ }^{\circ} \mathrm{C}$ and $+120^{\circ} \mathrm{C}$ and emissivity was set at 0.97 .

Static thermal images were taken at a $90^{\circ}$ angle at a distance of $1 \mathrm{~m} \pm 50 \mathrm{~cm}$ from the horse in accordance with recommendations from existing research (Stewart et al., 2005; Yarnell et al., 2013). Images were uploaded to the FLIR Quickreport analytical software and using a mouse interface the polygon analysis tool was placed over the area of the left and right semitendinosus muscle (Fig. 2) and maximum temperature extracted. Maximum temperature was chosen as existing work has stated that this is the most consistent

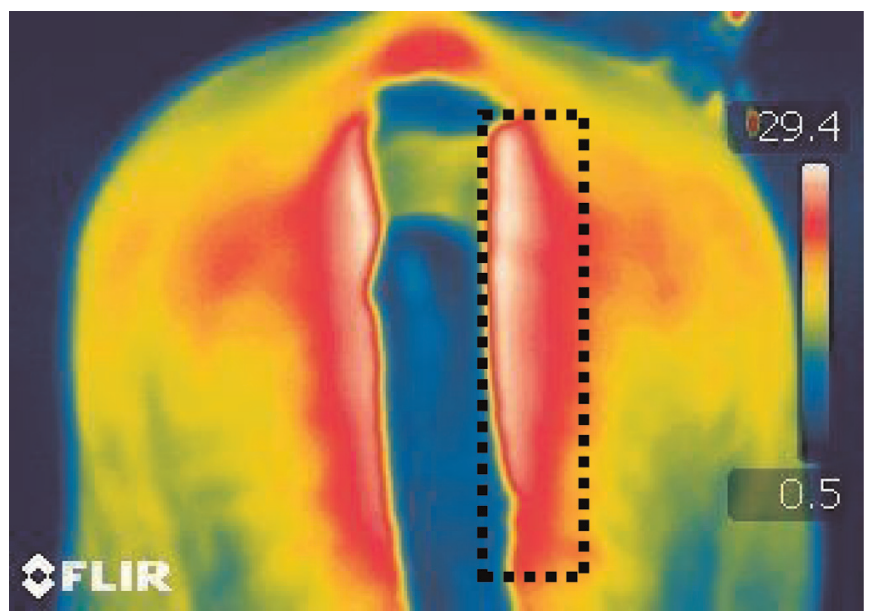

Fig. 2. Example thermal image of the equine hindquarters with area of the semitendinosus muscle highlighted. This figure shows an example thermal image of the equine hindquarters with thermal scale included on the right side of the image. The polygon analysis function has been utilised (dotted rectangular line on the right side of the image on the hindquarters) to highlight the area of the semitendinosus muscle which is the area from which maximum temperature was extracted. 
measure giving the least variance (Cook et al., 2006; Stewart et al., 2005).

\subsubsection{Infrared thermography standardisation}

In accordance with recommendations from Tunley and Henson (2004) a number of measures were put in place to standardise the thermal images captured. Horses were not ridden on any of the study days it was ensured that all horses were dry and clean one hour prior to their treadmill session. This was to prevent any heat generated through grooming of the coat to have dissipated before the study commenced. The horses tails were bandaged to ensure a clear view of the hind limbs and all boots and rugs were removed thirty minutes prior to treadmill exercise, again this was to allow any heat due to the insulating properties of the horses rugs to have dissipated before testing began. The room where the treadmill was housed was enclosed with a large front door that allowed natural light but not strong solar radiation to enter the building. In addition no artificial cooling methods were used on the horse and ambient temperature was monitored in the treadmill room. Whilst on the treadmill horses were not permitted to pass forward or backwards beyond the set boundaries of the treadmills glass viewing panel (Fig. 3). This was to ensure a consistent distance for image capture for all horses. All horses had at least five minutes to acclimatise to the water treadmill room before exercise commenced.

\subsection{Data analysis}

For each water height a mean surface temperature $\left({ }^{\circ} \mathrm{C}\right)$ was taken from left and right hind limb for each horse at each sampling time point. A mean surface temperature of all horses at each sampling time point was then calculated for each water height. The impact of water height on surface hind limb temperature was modelled using a mixed model approach ( $\mathrm{R}$ Package; nlme). Data from point 3 (immediately before warm up) to point 10 were included to model the whole exercise time period. Hind limb surface temperature (square root transformed) was included as the response variable with treatment (TD, PIP, CP) and time (centred) as predictors. To accurately model how temperature varied over time, the model was varied to include the quadratic, cubic and quartic terms for time and their interactions with treatment, the best fitting model was then selected using Akaike's information criterion (AIC).

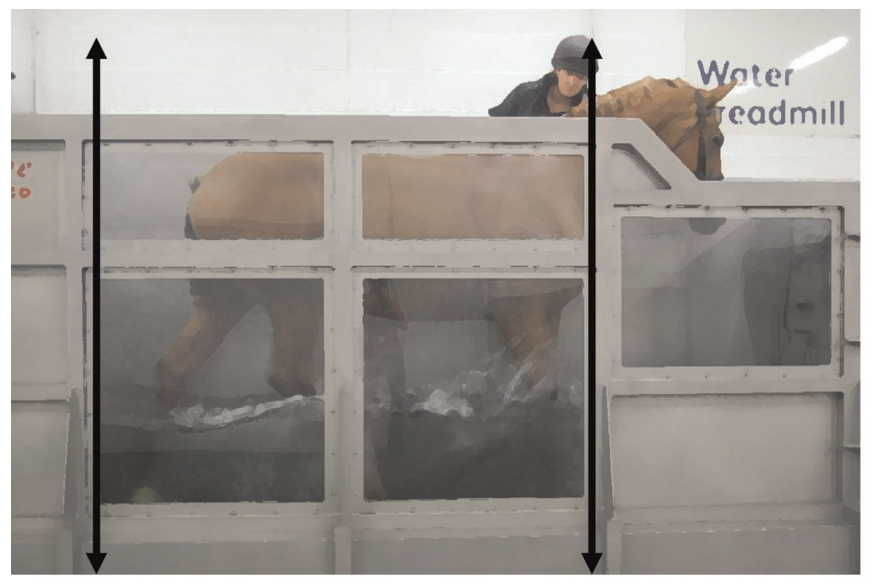

Fig. 3. A study horse working on the water treadmill. This figure shows one of the study horses exercising on the water treadmill with water at the height of the CP. The black arrows identify the points where the horse was not permitted to pass beyond (either forward or backwards in direction) in order to standardise distance that the thermal images were captured.
To further explore the three separate stages of the test (Warm up, main exercise, cool down) three further mixed effect models were ran with temperature as the response and time, treatment and their interaction as predictors. The first model included time points $3-4$, the second $4-8$ and the third $8-10$. These models were then restricted by removing non-significant effects in a backwards stepwise procedure until only significant effects remained in the model.

Horse ID was included in all analyses as a random effect to account for repeated measures. Significances for model effects were calculated using type III sums of squares. Normality assumptions for all models were checked by visualising residual plots. All statistical analyses were performed using R 3.0.2.

The change in surface temperature from sampling time point 3 to maximum temperature was calculated for each horse during each of the three treatments (TD, PIP, CP) Time point 3 was used as a base measure as prior to this all of the horses were subjected to identical exercise protocols (Table 1) and after time point 3 the exercise regime differed with regards to water height. A one way repeated measures ANOVA was conducted to investigate any differences in temperature change from point 3 to point 8 between treatments.

\section{Results}

One horse was required to be removed from the study, therefore, data is available for the seven remaining study horses.

Hind limb surface temperature in the region of the semitendinosus muscle increased at the onset of exercise and continued to rise until exercise was complete. This increase in temperature occurred at all three water heights (Fig. 4). Once exercise had ceased hind limb surface temperature began to decline and had returned to basal levels for all treatments at the final IRT sampling point.

The change in temperature over the whole exercise period was best modelled using the quartic function of time. This analysis demonstrated a significant difference in hind limb surface temperature in the region of the semitendinosus muscle between the different water heights during exercise on the water treadmill, (F2,

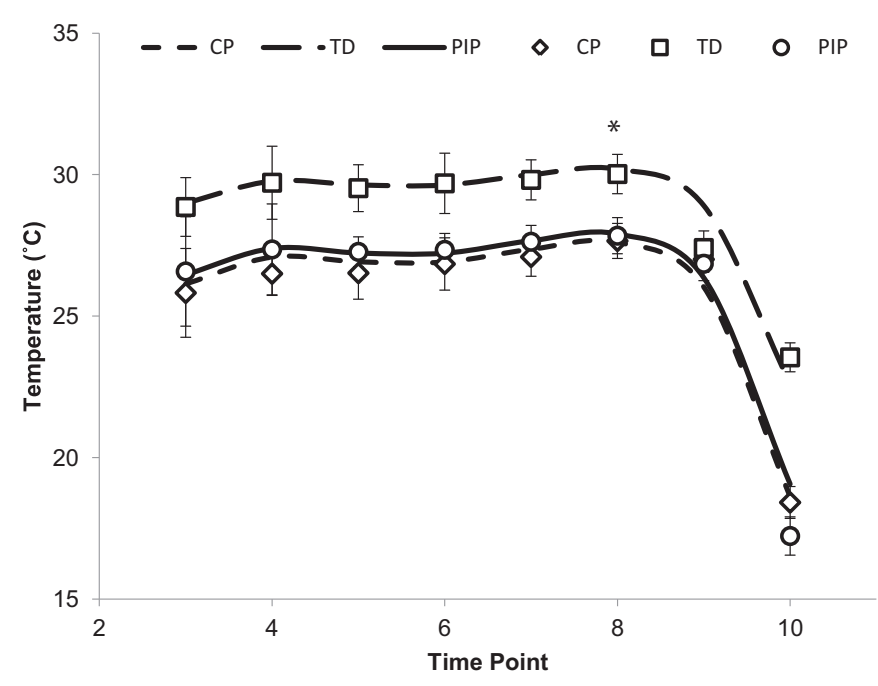

Fig. 4. Mean hind limb surface temperature (+standard error) over time during the three treatments: This figure shows the mean ( \pm standard error) hind limb surface temperature measured using IRT of the seven study horses whilst working on the water treadmill at each of the three water heights (TD, PIP and CP). The analysis was performed from sampling time point 3 as prior to this the protocol was identical for all treatments (PIP - circle, CP - diamond, TD - square). Lines show model fit. 
$155=38.96, P<0.0001$ ), but showed no difference in the pattern of change in temperature over time between treatments. Preplanned contrasts showed the difference in temperature was between TD and both $\mathrm{CP}$ and PIP treatments $(P<0.0001)$ with hind limb surface temperature being significantly warmer during the TD treatment when compared to both PIP and CP treatments. There was no significant difference in hind limb surface temperature between the PIP and CP treatments $(P>0.05)$.

When the model was broken down by stages, during the warm up stage there was no significant increase in temperature and no difference in rate of temperature change between treatments but there was a difference in absolute temperature between treatments $(F 2,33=8.79, P<0.0001)$. During the main exercise period there was also no significant change in rate of temperature change between or within treatments but there was again a difference in absolute temperature between treatments ( $F 2,96=32.85$, $P<0.0001)$. During the cool down period temperature also differed between treatments $(F 2,53=7.38, P<0.01)$ and there was a significant decrease in temperature $(\beta=-0.67 \pm 0.07, \quad F 1$, $53=97.27, P<0.0001$ ), during all three treatments but the rate of change did not differ between treatments. At all three time points planned contrast showed the difference in temperature was between TD and both PIP and CP with TD being warmer $(P<0.001)$, but not between PIP and CP $(P>0.05)$.

The maximum hind limb surface temperature recorded during the study was $30^{\circ} \mathrm{C}$ and this was observed at 15 min post-onset of exercise during the TD treatment. In addition maximum hind limb surface temperature was recorded at fifteen minutes post-onset of exercise for water at the height of the PIP and water at the height of the CP. This sampling time point was the final image taken before exercise ceased.

There was a greater increase in surface hind limb temperature for all horses during the $\mathrm{CP}$ and PIP treatments when compared to the TD treatment (Fig. 5). However, the results of a one way repeated measures ANOVA showed this difference to be not significant $(F 2,12=0.56, P=0.586)$.

\section{Discussion}

Hind limb surface temperature in the region of the semitendinosus muscle increased at the onset of exercise in all three treatments (TD, PIP and CP) suggesting that heat produced by muscle contraction (Shepherd, 1982) and increases in local blood

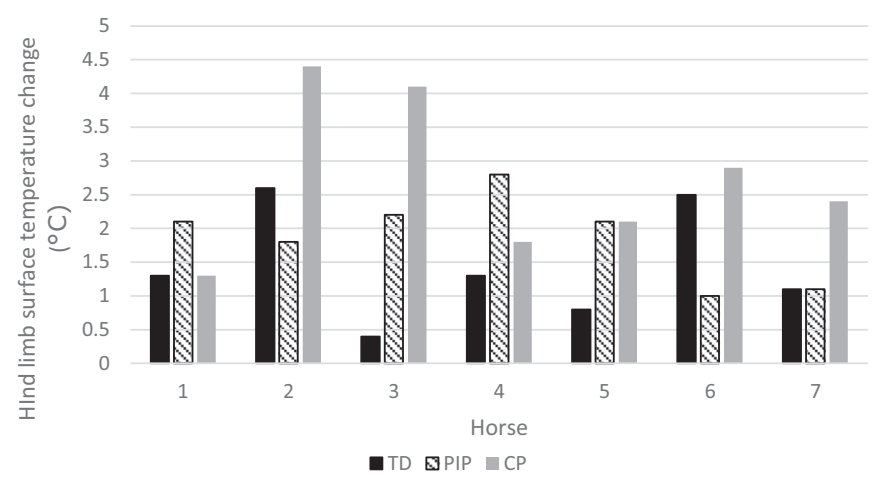

Fig. 5. Change in hind limb surface temperature $\left({ }^{\circ} \mathrm{C}\right)$ from pre warm up until maximum temperature. This figure shows the change in hind limb surface temperature $\left({ }^{\circ} \mathrm{C}\right.$ ) from time point 3 (immediately before warm up) until maximum temperature for each horse $(n=7)$ during each of the three treatments which were treadmill dry (TD), Water at height of the proximal interphalangeal joint (PIP) and water at the height of the carpus (CP). The maximum temperature recorded was at time point 8 (immediately before exercise ceased) for all horses in all treatments. flow (Eddy et al., 2001) in order to meet the metabolic demands of the working tissues can be identified using IRT.

Surface temperature of the hind limbs underwent a greater increase from pre warm up levels during exercise in water than when horses were exercised without water although this was not significant $(P=0.58)$.

No significant differences in rate of temperature change was identified within or between each treatment with all three exercise protocols. This suggests that surface temperature gradually increased and the rate of temperature increase was not dependent or effected by water height within the treadmill.

Hind limb surface temperature had started to increase before exercise commenced (at sampling point 2). This is likely to be due to increased muscle activity and associated alterations in blood flow as a result of the horse walking to the treadmill room from its stable and walking up the incline onto the treadmill. This suggests that muscle contraction and associated blood flow alterations to support the increased muscle activity results in a rapid change in surface temperature even as a result of low intensity exercise. Hind limb surface temperature did continue to increase once exercise commenced and until exercise ceased but this increase was gradual with no significant rise between any sampling time points.

Surface temperature gradually increased throughout all three exercise sessions with a maximum temperature of $30^{\circ} \mathrm{C}$ recorded during the TD treatment. The presence of water in the treadmill may have resulted in a cooling effect upon the circulation and subsequently may have caused the lower surface temperatures recorded during the PIP and $\mathrm{CP}$ treatments. However, water did not come into direct contact with the skin surface in the area of the semitendinosus muscle and only reached the level of the carpus. In addition, basal surface temperature was higher during the TD treatment when compared to the PIP and CP treatments. Maximum surface temperatures for all three treatments were recorded at $15 \mathrm{~min}$ post-onset of exercise $\left(\mathrm{PIP}=27.9^{\circ} \mathrm{C}\right.$, $\mathrm{CP}=27.7^{\circ} \mathrm{C}$ ) which was the last thermal image captured before exercise ceased. Surface temperature then began to decline and had returned to basal levels for all three treatments at three minutes post-exercise. This decline in surface temperature was significant in all three treatments $(P<0.0001)$ with no difference in rate of change between treatments. The significant decrease in temperature during the cool down period may have been due in part to the draining of the water (and therefore a reduction in resistance); however, the significant decrease in temperature was also apparent during the TD treatment where no resistance was present. Therefore, it is likely that a combination of the removal of resistance of water and termination of exercise itself contributed to the rapid cooling of the surface temperature of the hind limbs. This also highlights that the rate of decrease in surface temperature was not dependent on the maximum temperature reached as surface temperature had returned to below basal levels in all three treatments at the final IRT sampling point.

Horses sweat to dissipate heat through evaporation of moisture from the skin surface and this could have contributed to rapid cooling post-exercise. However, the study horses walked at a comfortable pace for fifteen minutes and had taken part in treadmill work in the weeks preceding this investigation, therefore, they were of sufficient fitness for this type of exercise. As a result, none of the study horses displayed obvious signs of sweating and airflow was minimal due to the stationary nature of treadmill exercise and the absence of artificial cooling.

The gradual increase in temperature throughout the exercise sessions supports the findings of Lovell et al. (2006) who found a positive correlation between muscle temperature and exercise duration in horses, with muscle temperature gradually increasing up to $38.8( \pm 0.5){ }^{\circ} \mathrm{C}$. The higher temperature compared to our 
study findings may be a reflection of the temperature collection method of muscle biopsy rather than surface temperature.

As exercise intensity increases so does the temperature of working muscle (Geor et al., 2000; McGowan et al., 2002). Therefore, the larger increase in surface temperatures recorded with the addition of water during this study suggest that the increased resistance provided by the water resulted in higher muscle activity (contraction of tissue and alterations in bloodflow) that was able to be detected using IRT. However, the height of the water did not significantly alter the surface hind limb temperature when a comparison was made between the PIP and the CP.

Scott et al. (2010) found that increasing water height from the foot up to the carpal joint in horses on a standard treadmill altered the stride frequency of those horses. This biomechanical change is not reflected physiologically in the present study; however, it may be that IRT was unable to detect an altered muscle workload between the two water heights. It could also be that no significant difference in hind limb surface temperature between the PIP and $\mathrm{CP}$ is due to increased muscular activity and blood flow changes in both treatments caused by different aspects of water treadmill exercise. The increased stride length and decreased stride frequency reported by Scott et al. (2010) with water at the height of the carpus is due to the resistance of water and therefore a higher workload for the muscles involved. Whereas, when horses were exercised in water at the height of the PIP this required a higher flight arc (stepping motion) from the horse, therefore, greater hind limb flexion which also resulted in increased muscle contraction and changes in blood flow. Both scenarios result in a higher workload for the muscles, therefore, increased heat dissipation that was identified with IRT. This has implications for equine professionals who must select specific parameters including water height when devising training and rehabilitation programmes.

Further work is needed to investigate the effect of other water heights used in water treadmill exercise (fetlock, shoulder, hock) on hind limb surface temperature and whether IRT can detect potential differences, in addition to other muscle groups utilised in equine movement. It would be interesting to see whether an increased length of time exercising at the two water heights investigated in this study would result in a clear difference in hind limb surface temperature when measured using IRT. An additional area of further study would be to investigate whether significantly warming or cooling of the water within the treadmill has any physiological effect on surface temperatures during exercise. This information could then be used to further develop exercise programmes with regards to target muscle groups and timing of activity.

It must be considered that surface temperature changes observed during this study could be the result of changes in other anatomical structures beneath the surface of the skin. However, this seems unlikely as the semitendinosus muscle is a superficial structure that is able to be palpated by hand and changes in thermal patterns on the skin surface correspond to its location. Redaelli et al. (2014) state that the skin overlying muscles is subject to an increase in temperature during muscular activity largely due to changes in vascularity and it has been shown that this particular muscle group is active during treadmill exercise and contributes to the stepping motion involved (Robert et al., 2000).

Kastelic et al. (1996) found that abrupt changes in ambient temperature resulted in confounding results during use of IRT and the authors suggest that moderate to cool temperatures of between 5 and $15{ }^{\circ} \mathrm{C}$ are ideal to capture thermal images. No abrupt fluctuations were recorded during this study and the recorded mean ambient temperature was $9.7^{\circ} \mathrm{C}$ which falls within these recommended parameters.

Infrared thermography cannot measure actual muscle temperature but it can give an indication of the underlying tissue metabolism and changes in blood flow which reflects the working muscle in horses undergoing water based rehabilitation or training. Water treadmill exercise may be beneficial to horses as it can provide the resistance of water with the advantage of controlled parameters such as speed and incline that are not possible to control when swimming horses in a hydrotherapy pool and IRT has highlighted some interesting findings during this study that equine trainers and therapists will find useful including information of warm up and cool down timings and the increased resistance but minor difference in impact upon muscle activity and blood flow changes in the two water heights studied.

The potential impact of IRT on the equine industry and its further possible applications are very encouraging. IRT could be used as a rapid, non-invasive screening tool to assess surface temperature surrounding other important muscle groups involved in equine movement and performance. In addition IRT could be used during training programmes to ensure muscles are working symmetrically and indicate which terrains or training programmes results in an increase in muscle workload. IRT could also indicate whether target muscle groups are responding to applied workload and which muscle groups are being worked with specific exercise regimes. From a therapeutic point of view IRT could allow monitoring of surface temperature as an indirect measure of muscle activity and associated changes in blood flow in surgical patients who are not suitable candidates for invasive temperature probes but would benefit from hydrotherapy. IRT could monitor these parameters and how they change over time during a rehabilitation programme and provide important information to physiotherapists regarding length and intensity of treatment.

\section{Conclusion}

This study has demonstrated that IRT can detect surface temperature change associated with underlying muscle activity and associated changes in blood flow in horses. The assessment of temperature change as a result of the underlying physiological response to exercise in an aquatic environment has not been carried out to date in horses. Further work is now required to explore further applications of IRT and its growing popularity within the equine industry.

\section{Acknowledgements}

The authors wish to thank the equine therapy centre staff at Bishop Burton College for the provision of their horses, thermal camera and water treadmill. Thanks to David Jukes at Nottingham Trent University for his assistance with production of artwork and thank you to Cassie White for Biomechanical and exercise physiology based advice.

\section{References}

Cena, K., Clark, J.A., 1973. Thermographic measurements of the surface temperatures of animals. J. Mammal. 54, 1003-1007.

Crook, T., Wilson, A., Hodson-Tole, E., 2010. The effect of treadmill speed and gradient on equine hindlimb muscle activity. Equine Vet, J. 42 (38), 412-416.

Eddy, A., Van Hoogmoed, L., Snyder, J., 2001. The role of thermography in management of equine lameness. Vet. J. 162 (10), 172-181.

Geor, R., McCutcheon, L., Hinchcliff, K., 2000. Effects of warm-up intensity on kinetics of oxygen consumption and carbon dioxide production during highintensity exercise in horses. Am. J. Vet. Res. 61 (6), 638-645.

Kastelic, J.P., Cook, R.B., Coulter, G.H., Wallins, G.L., Entz., T., 1996. Environmental factors affecting measurement of bovine scrotal surface temperature with infrared thermography. Anim. Reprod. Sci. 41, 153-159. 
King, M.R., Haussler, K.K., Kawcak, C.E., McIlwraith, C.W., Reiser, R.F., 2012. Mechanisms of aquatic therapy and its potential use in managing equine osteoarthritis. Equine Vet. Educ. 10 (1), 2042-3292.

Linder, A., Wurm, S., Beuttler, J., Hermann, H., Sasse, L., 2003. Effect of water height on biochemistry and heart rate of horses exercising on a treadmill submerged in water. Equine Nutr. Physiol. 18 (1), 204-206.

Lovell, D.K., Reid, T.A., Rose, R.J., 2006. Effects of maximal exercise on equine muscle changes in metabolites, pH and temperature. Equine Exerc. Physiol., 312-319.

McGowan, C., Fordham, T., Christley, R., 2002. Incidence and risk factors for extertional rhabdomyolysis in thoroughbred racehorses in the United Kingdom. Vet. Res. 151 (1), 623-626.

McGreevy, P., Warren-Smith, A., Guisard, Y., 2012. The effect of double bridles and jaw clamping crank nosebands on temperature of eyes and facial skin of horses. J. Vet. Behav. 7 (3), 142-148.

McKenzie, E., 2005. Exercise associated muscle injury in horses. Sport. Inj. Horses Man 21, 1-6.

Matsuo, T., Watanabe, K., Takahashi, T., Sakamoto, K., Yamamoto, K., 2006. Application of thermography for evaluation of mechanical load on the muscles of upper limb during wheelchair driving. J. Biomech. 39 (1), 537.

Miyoshi, T., Shirota, T., Yamamoto, S.I., Nakazawa, K., Akai, M., 2004. Effect of the walking speed to the lower limb joint angular displacements, joint moments and ground reaction forces during walking in water. Disabil. Rehabil. 26 (1), 724-732.

Nakayama, K., Got, S., Karaoke, K., Nakamura, K., 2005. Decrease in nasal temperature of rhesus monkeys (Macaca mulatto) in negative emotional state. Physiol. Behav. 84 (5), 783-790.

Parrott, R.F., Lloyd, D.M., Brown, D., 1999. Transport stress and exercise hyperthermia recorded in sheep by radiotelemetry. Anim. Welf. 8 (1), 27-34.

Pusey, A., Brooks, J., Jenks, A., 2011. Osteopathy and the Treatment of Horses. WileyBlackwell, United Kingdom.

Redaelli, V., Bergero, D., Zucca, E., Ferrucci, F., Nanni Costa, L., Crosta, L., Luzi, F., 2014. Use of thermography techniques in equines: principles and applications. J. Equine Vet. Sci. 34, 345-350.

Rivero, J.L.L., Piercy, J., 2008. Proceedings of the Conference on Equine Sports Medicine and Science. Wageningen Academic, Netherlands.
Robert, C., Valette, J., Denoix, J., 2000. The effects of treadmill inclination and speed on the activity of two hindlimb muscles in the trotting horse. Equine Vet. J. 32 (4), 312-317.

Schaefer, A.L., Matthews, L.R., Cook, N.J., Webster, J., Scott, S.L., 2002. Novel noninvasive measures of animal welfare. In: Proceedings of Animal Welfare and Behaviour, from Science to Solution Joint NAWAC/ISAE Conference, Hamilton, New Zealand.

Scott, R., Nankervis, K., Stringer, C., Westcott, K., Marlin, D., 2010. The effect of water height on stride frequency, stride length and heart rate during water treadmill exercise. Equine Vet. J. 42 (38), 662-664.

Shepherd R.J., Physiology and Biochemistry of exercise, 1982, Praegar, New York.

Simon, E.L., Gaughan, E.M., Epp, T., Spire, M., 2006. Influence of exercise on thermographically determined surface temperatures of thoracic and pelvic limbs in horses. J. Am. Vet. Med. Assoc. 229 (12), 1940-1944.

Stewart, M., Webster, J.R., Schaefer, A.L., Cook, N.J., Scott, S.L., 2005. Infrared thermography as a non-invasive tool to study animal welfare. Anim. Welf. 14, 319-325.

Tokuriki, M., Ohtsuki, R., Kai, M., Hiraga, A., Oki, H., Miyahara, Y., Aoki, O., 1999. EMC Activity of the muscles of the neck and forelimbs during different forms of locomotion. Equine Exerc. Physiol. 5 (Suppl. 30), 231-234.

Tunley, B., Henson, F., 2004. Reliability and repeatability of thermographic examination and the normal thermographic image of the thoracolumbar region in the horse. Equine Vet. J. 36 (4), 306-312.

Turner, T.A., 2001. Diagnostic thermography. Vet. Clin. N. Am. Equine Pract. 17 (1) 95-113.

Van de Graaffe, K., Fox, S., Thouin, L., 1999. Concepts of Human Anatomy and Physiology. McGraw-Hill, United States of America.

Von Schweinitz, G., 1999. Thermographic diagnostics in equine back pain. Vet. Clin. N. Am. Equine Pract. 15 (1), 161-177.

Voss, B., Mohr, E., Krzywanek, H., 2002. Effects of aqua-treadmill exercise on selected blood parameters and on heart-rate variability of horses. J. Am. Vet. Med. Assoc. 49 (1), 137-143.

Yarnell, K., Hall., C., Billet, E., 2013. An assessment of the aversive nature of an animal management procedure (clipping) using behavioural and physiological measures. Physiol. Behav. 118, 32-39. 